\title{
Aprendizaje basado en problemas en el diseño de prácticas de laboratorio online
}

\section{Beatriz Jurado-Sánchez* y Ana María Díez-Pascual}

Universidad de Alcalá, Facultad de Ciencias, Departamento de Química Analítica, Química Física e Ingeniería Química, Ctra. Madrid-Barcelona, Km. 33.600, 28871 Alcalá de Henares, Madrid, España (beatriz.jurado@uah.es; am.diez@uah.es)

\section{\$EWWDW}

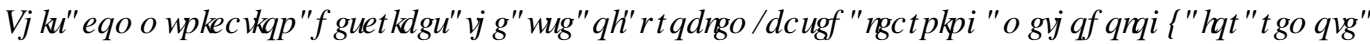

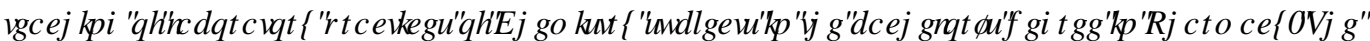

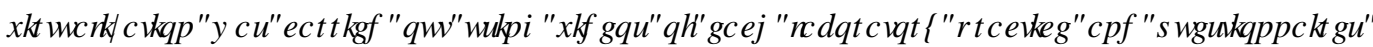

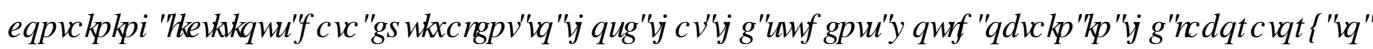
FDU

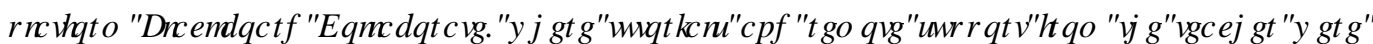
FDUHGTRXWXRIVROHWKHSUREGP VULUHGD

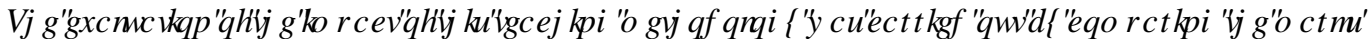

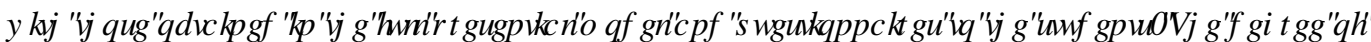

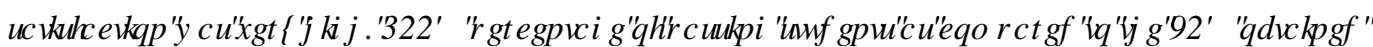

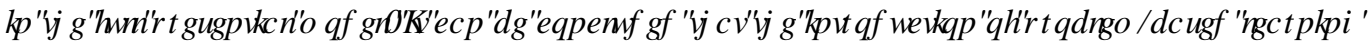

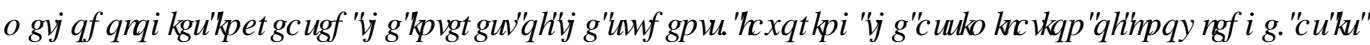

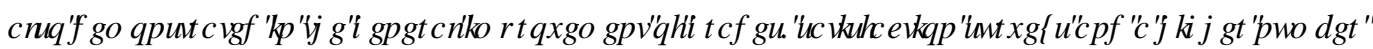

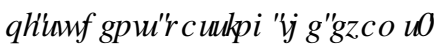

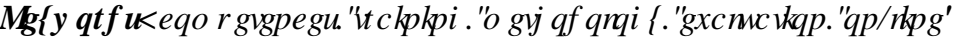

\section{HXP HQ}

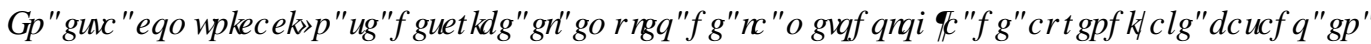

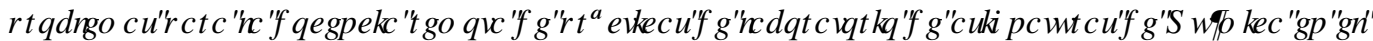

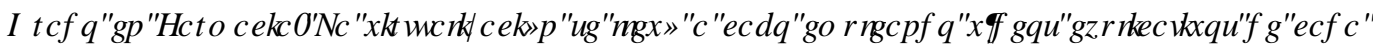

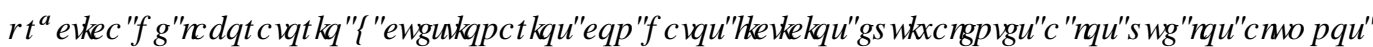

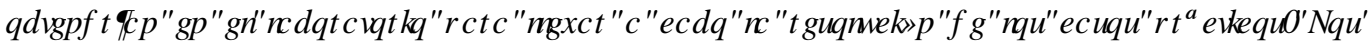

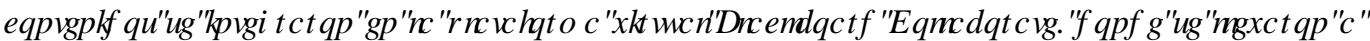

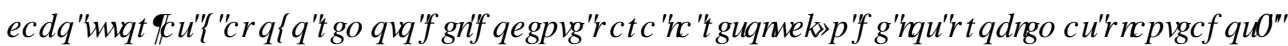

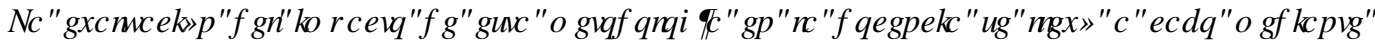

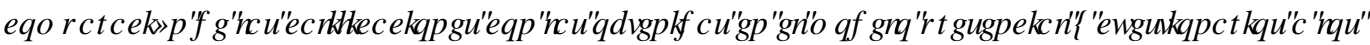

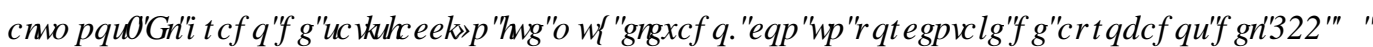

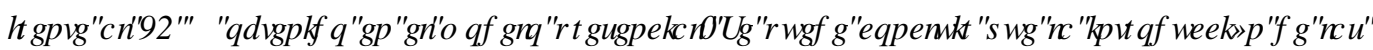

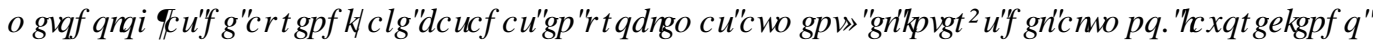

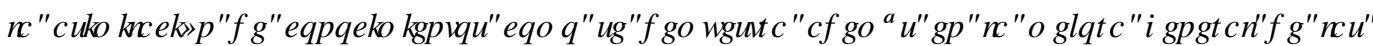

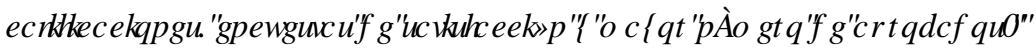

3 DOEUVFOVH 


\section{Introducción y objetivos}

La pandemia de COVID-19 y la situación de confinamiento en marzo de 2020 supuso una la paralización de la docencia y a la actividad presencial en la Universidades. En el contexto de las clases teóricas, las plataformas virtuales facilitaron la impartición de docencia en éstas mediante videoconferencias y presentaciones pregrabadas (Bao, W, 2020; Hodges, et al. 2020). Sin embargo, las prácticas de laboratorio (en especial en las áreas de ciencias) requieren una participación activa del alumnado en el proceso de enseñanza aprendizaje para la adquisición de competencias en el manejo activo de material de laboratorio o la resolución de problemas en el campo de la química, control farmacéutico, etc (Llorens-Molina, M., 2010).

Esta comunicación surge de nuestra experiencia docente en el curso 2019/2020 en la docencia de la asignatura “Analytical Techniques” del segundo curso del Grado en Farmacia de la Universidad de Alcalá. La asignatura presenta varias problemáticas inherentes que dificultan el proceso enseñanza-aprendizaje. En primer lugar, es la primera vez que los alumnos reciben conocimientos acerca de la materia (Química Analítica), con los que no están familiarizados. En segundo lugar, se trata de una asignatura de duración anual con 6 bloques temáticos y además 2 sesiones de prácticas de laboratorio de una duración estimada, aproximadamente dos semanas cada una. En tercer lugar, la asignatura se imparte en inglés, un idioma en el que muchos alumnos no presentan un dominio adecuado, añadiendo aún más dificultad si cabe para la asimilación de conceptos. Todo ello redunda además en una falta de interés por parte del alumnado, que en ocasiones se siente frustrado. Como metodologías docentes se utiliza la clase magistral y las prácticas de laboratorio. Estas prácticas se realizan en dos períodos presenciales de 6 días de duración cada uno y que comprenden 6 prácticas experimentales, con un examen final práctico. La situación del confinamiento supuso la imposibilidad de realizar presencialmente la segunda sesión de prácticas de laboratorio, por lo que la docencia presencial tradicional tuvo que transformarse en un modelo de enseñanza remota de emergencia.

El aprendizaje basado en problemas es una herramienta muy adecuada en el proceso de enseñanzaaprendizaje ya que permite aplicar el aprendizaje activo a la resolución de problemas del mundo real como los planteados en las prácticas anteriormente mencionadas (Barell, J, 1999; Arpí, et al, 2012; Gil-Galván, 2018). Este hecho es muy adecuado para suplir las carencias del formato on-line en el contexto de las prácticas de laboratorio y permite el trabajo colaborativo de grupos de estudiantes. El docente queda en un segundo plano, con un papel orientativo, pero con la capacidad de motivar al alumnado para que participe de forma más activa y comprenda la finalidad de las prácticas (Martinez, C., et al, 2020).

El objetivo de esta comunicación es compartir nuestra experiencia en la virtualización de las prácticas de laboratorio de Analytical Techniques y la integración de la metodología de aprendizaje en problemas. Para ello se empleó la herramienta Blackboard Collaborate integrada en la plataforma virtual de la Universidad de Alcalá en la que se integraron vídeos explicativos de cada práctica de laboratorio. Se elaboró un cuestionario con preguntas relacionadas con los experimentos y datos ficticios equivalentes a los que los alumnos obtendrían en el laboratorio para llevar a cabo la resolución del caso/práctica de laboratorio. Para la evaluación del proceso enseñanza-aprendizaje, se elaboró una hoja de rúbrica para la calificación de los informes y un examen final. La evaluación del impacto de esta metodología y el nuevo enfoque en la docencia se llevó a cabo mediante comparación de las calificaciones con las obtenidas en el modelo presencial y cuestionarios a los alumnos.

\section{Desarrollo de la innovación}

La virtualización de los contenidos se llevó a cabo en la herramienta Blackboard Collaborate integrada en la plataforma virtual de la Universidad de Alcalá. Se virtualizaron 6 prácticas de laboratorio descritas en la Guía docente de la asignatura, como se describen a continuación: 
- Analysis of sulphates by turbidimetry

- Analysis of sodium and potassium by flame photometry

- Conductimetric titration of acids

- Separation of compounds by high performance liquid chromatography

- Analysis of mixtures by molecular absorption spectrophotometry

- Determination of paracetamol in pharmaceutical products by cyclic voltammetry

La Tabla 1 describe una planificación de la metodología propuesta para llevar a cabo la virtualización de las prácticas y la integración de las metodologías de aprendizaje basado en problemas. Se proporciono el cuaderno de laboratorio a los alumnos junto con videos explicativos del desarrollo de las prácticas. Para cada práctica, se diseñó un cuestionario con preguntas previas y datos numéricos similares a los que obtendrían en el laboratorio con el objetivo de resolver la problemática planteada en las prácticas de laboratorio. La Figuras 1 y 2 muestran ejemplos representativos del cuestionario de laboratorio correspondiente a las prácticas.

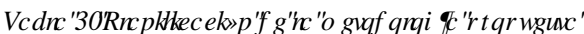

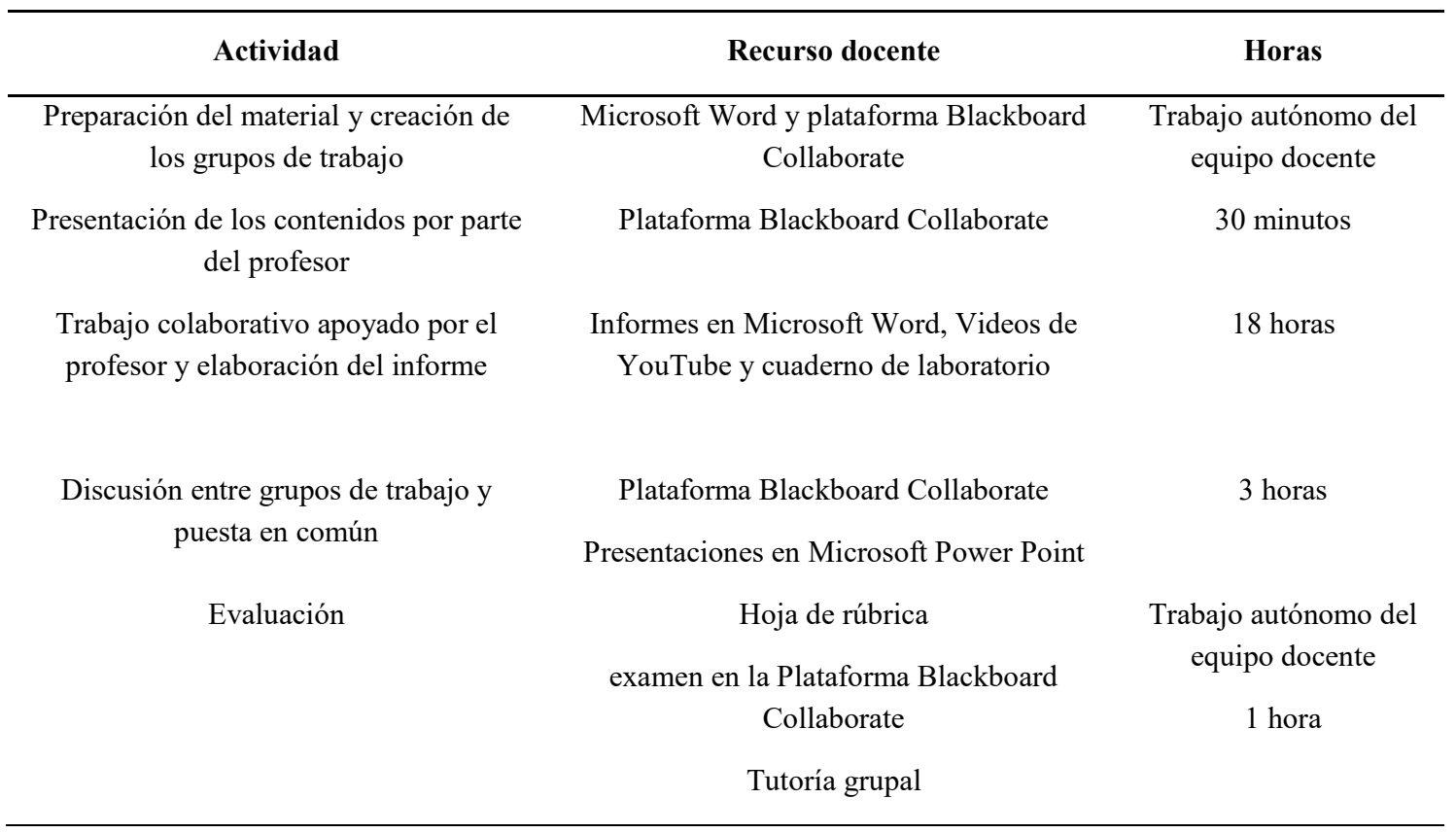

]

Tras llevar a cabo la virtualización, los alumnos se organizaron en grupos y se les proporciono un cronograma con una planificación temporal para que realizasen la visualización de los videos, lectura del cuaderno de laboratorio y resolución de los informes en un período de 7 días. Tanto los alumnos como los profesores se conectaron a la plataforma en el tiempo establecido en el cronograma. El último día se celebró una tutoría grupal orientativa para resolver dudas y cuestiones suscitadas por los alumnos durante el desarrollo de las prácticas. 
11. DETERMINATION OF PARACETAMOL IN PHARMACEUTICAL PRODUCTS BY CYCLIC VOLTAMMETRY. REPORT

PRELIMINARY QUESTIONS

1. Indicate the parameter(s) that is/are monitored in a cyclic voltammetry and draw the shape of the applied potential as a function of time.

2. Calculate the amount of acetaminophen (in $\mathrm{mg}$ ) needed to prepare $5 \mathrm{~mL}$ of a $1 \times 10^{-4}$ M solution in buffer.

MW, acetaminophen $=151.16 \mathrm{~g} / \mathrm{mol}$
11.1.- Report the values for the cathodic peak potential $\mathrm{E}_{\mathrm{po}}$ the anodic peak potential $E_{p a}$, the cathodic peak current $i_{p o}$ and the anodic peak current $i_{p a}$ from the cyclic voltammogram obtained in part 1.

Assume that you perform the $\mathrm{CV}$ measurements, obtaining the following cyclic voltammogram:

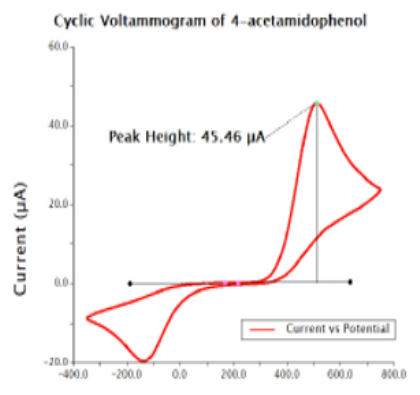

Potential (mV)

11.2. Represent the calibration plot and obtain the calibration graph and the analytical figures of merit.

Assume that you have prepared all the standard solutions and measured the peak height of the cathodic peak. The data that you should obtain are reported in the following table:

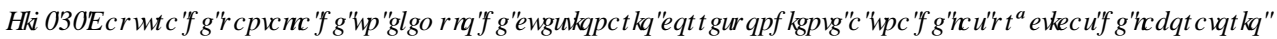

[1

\section{PHESENIATION Of THE RESULTS}

8. Plot the conductance obtained versus the volume of $\mathrm{NaOH}$ added for the three condactometric titration.

82. Calculate the equivalence point in each titration curve.

1. Titration of $\mathrm{HC}$ with $\mathrm{NaOH}$.

Assume that you performa the titration of a $50.00 \mathrm{ml}$ of $\mathrm{BCL}$, problems solution with $0.100 \mathrm{M}$ $\mathrm{NaOH}$ sobution The following data of conductance vs. volume of $\mathrm{NaOH}$ added werectoined. With the data calculate the equivivient poitn.

\begin{tabular}{|c|c|}
\hline $\mathbf{V}_{\mathbf{w a}}(\mathrm{mL})$ & $\mathbf{C}(\boldsymbol{\mu s})$ \\
\hline 1 & 706.8 \\
\hline 2 & 659.9 \\
\hline 3 & 613.8 \\
\hline 4 & 569.6 \\
\hline 5 & 521.2 \\
\hline 6 & 475.6 \\
\hline 7 & 426.7 \\
\hline 8 & 379.7 \\
\hline 9 & 334.6 \\
\hline 10 & 294.3 \\
\hline 11 & 250.8 \\
\hline 12 & 239.1 \\
\hline 13 & 265.2 \\
\hline 14 & 301.8 \\
\hline 15 & 339.5 \\
\hline 16 & 347.4 \\
\hline 17 & 413.3 \\
\hline 18 & 447.4 \\
\hline 19 & 406.7 \\
\hline 20 & 523.1 \\
\hline
\end{tabular}

The plot obtained in excet by represeating volume of $\mathrm{NaOH}$ vs. conductance is as follows:

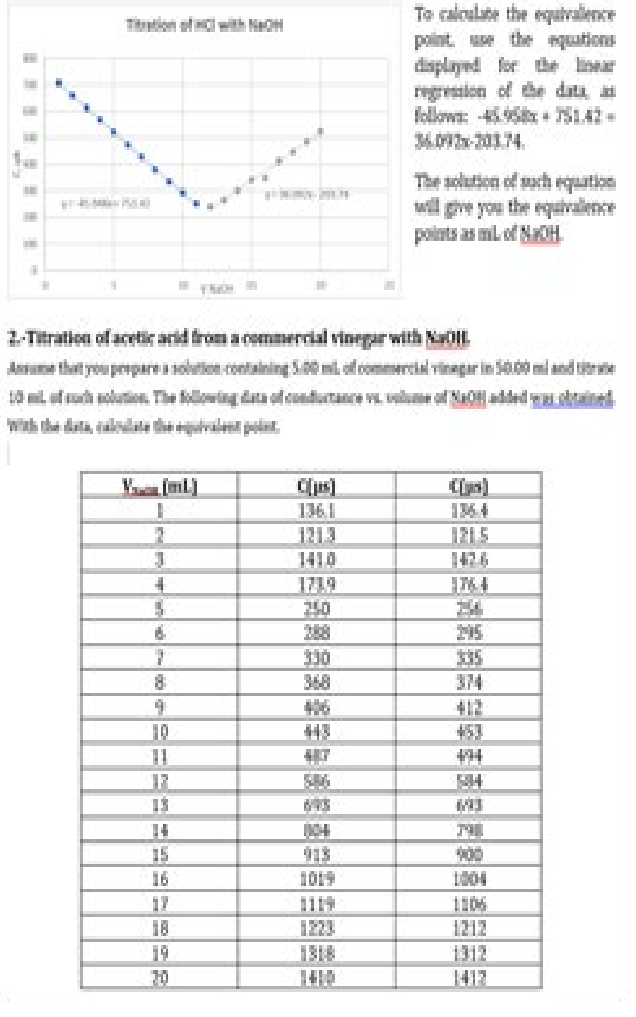

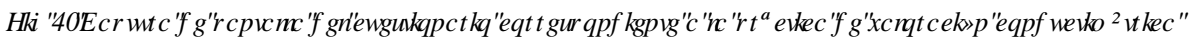

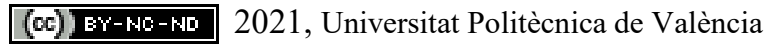

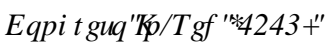


Para llevar a cabo la evaluación, los alumnos hicieron la entrega de la actividad a través de la Plataforma, a la que se le asignó una calificación numérica empleando una hoja de rúbricas (ver Figura 3), que supuso un $40 \%$ de la calificación total de las prácticas. Como puede observarse, la Hoja de Rúbricas se diseñó dando especial importancia a la integración del aprendizaje colaborativo y la participación del alumno.

\begin{tabular}{|c|c|c|c|c|}
\hline & Mal (0-2) & Regular (3-5) & Bien (5-8) & Muybien (9-10) \\
\hline $\begin{array}{l}\text { ASISTENCIA Y } \\
\text { PARTICPACIÓN EN LAS } \\
\text { SESIONES (10\%) } \\
\text { PREGUNTAS PREMAS } \\
(45 \%)\end{array}$ & $\begin{array}{l}\text {-No asiste. } \\
\text {-Falta a alguna sesión y no } \\
\text { lo justifica. } \\
\text {-No participa en clase. } \\
\text {-No responde a ninguna } \\
\text { pregunta } \\
\text {-Responde de forma } \\
\text { demasiado extensa pero con } \\
\text { Un concepto erróneo } \\
\text {-No justifica las respuestas }\end{array}$ & $\begin{array}{l}\text {-No asiste. } \\
\text {-Falta a alguna sesión y no } \\
\text { lo justifica. } \\
\text {-No participa en clase. } \\
\text {-Responde bien a una } \\
\text { pregunta } \\
\text {-Da una respuesta } \\
\text { demasiado extensa con un } \\
\text { concepto es parcialmente } \\
\text { erróneo } \\
\text {-No justifica las respuestas }\end{array}$ & $\begin{array}{l}\text {-No asiste. } \\
\text {-Falta a alguna sesión y no } \\
\text { lo justifica. } \\
\text {-No participa en clase. } \\
\text {-Responde bien a una } \\
\text { pregunta y parcialmente a } \\
\text { otra } \\
\text {-Da una respuesta concisa, } \\
\text { aunque el concepto es } \\
\text { parcialmente enóneo } \\
\text {-Justifica las respuestas } \\
\text { aunque no con ideas propias }\end{array}$ & $\begin{array}{l}\text {-Responde correctamente } \\
\text { todas las preguntas } \\
\text {-La respuesta es concisa } \\
\text { pero adecuada } \\
\text {-Justifica debidamente la } \\
\text { respuesta con ideas propias }\end{array}$ \\
\hline $\begin{array}{l}\text { RESOLUCIÓN Y } \\
\text { DISCUSÍN DE } \\
\text { PROBLEMAS NUMÉRICOS } \\
(45 \%)\end{array}$ & $\begin{array}{l}\text {-No desamolla bien los } \\
\text { problemas } \\
\text {-No da el resultado correcto } \\
\text { ni aproximado } \\
\text {-No expresa bien el } \\
\text { resultado en términos de } \\
\text { cifras significativas y error } \\
\text {-Comete errores en los } \\
\text { factores de divción } \\
\text {-No discute el resultado }\end{array}$ & $\begin{array}{l}\text {-Desamolla correctamente el } \\
50 \% \text { de los problemas } \\
\text {-Proporciona un resultado } \\
\text { aproximado aunque muy } \\
\text { cercano al correcto } \\
\text {-No expresa bien el } \\
\text { resultado en términos de } \\
\text { cifras significativas o error } \\
\text {-Comete errores en alguin } \\
\text { factor de dilución } \\
\text {-No discute el resultado }\end{array}$ & $\begin{array}{l}\text {-Desamola comectamente el } \\
75 \% \text { de los problemas } \\
\text {-Proporciona el resultado } \\
\text { comecto } \\
\text {-Expresa bien el enror del } \\
\text { resultado y las cifras } \\
\text { significafivas } \\
\text {-No comete enrores en los } \\
\text { factores de dilución } \\
\text {-Discute el resultado aunque } \\
\text { no con ideas propias }\end{array}$ & $\begin{array}{l}\text {-Desamolla correctamente el } \\
100 \% \text { de los problemas } \\
\text {-Proporciona el resultado } \\
\text { correctb } \\
\text {-Expresa bien el error del } \\
\text { resultado y las cifras } \\
\text { significativas } \\
\text {-No comete enrores en los } \\
\text { factores de dilución } \\
\text {-Discute el resultado con } \\
\text { ideas propias }\end{array}$ \\
\hline $\begin{array}{l}\text { PRESENTACIÓN DE } \\
\text { CUADERNO Y } \\
\text { PUNTUAUDAD EN LA } \\
\text { ENTREGA } \\
(10 \%)\end{array}$ & $\begin{array}{l}\text {-La presentación del } \\
\text { cuademo no es clara, } \\
\text { algunas fómulas son } \\
\text { iegibles } \\
\text {-En los problemas solo da el } \\
\text { resultado final } \\
\text {-Entrega tarde la actividad } \\
\text { (más de } 3 \text { dias de retraso) }\end{array}$ & $\begin{array}{l}\text {-La presentación del } \\
\text { cuademo no es clara, } \\
\text { aunque las fórmulas con } \\
\text { legbles } \\
\text {-En los problemas da el } \\
\text { resultado fral y proporciona } \\
\text { parcialmente los cálculos } \\
\text {-Entrega tarde la actividad } \\
\text { (menos de } 3 \text { dias de retraso) }\end{array}$ & $\begin{array}{l}\text {-La presentación del } \\
\text { cuademo es clara y legible, } \\
\text { aunque demasiado extensa } \\
\text {-En los problemas } \\
\text { proporciona los cálculos y el } \\
\text { resultado final } \\
\text {-Entrega tarde la actividad } \\
\text { (menos de dos dias de } \\
\text { retraso) }\end{array}$ & $\begin{array}{l}\text {-La presentación del } \\
\text { cuademo es clara, concisa y } \\
\text { legible } \\
\text {-En los problemas } \\
\text { proporciona los cálculos y el } \\
\text { resultado final } \\
\text {-Entrega la actividad en el } \\
\text { plazo exigido }\end{array}$ \\
\hline
\end{tabular}

) $L J \square \square+R N D G L E U F D H P S C D O D H Q D H D O D F Y Q]$

El 60 \% restante de la calificación se asignó a un examen integrado en la Plataforma Blackboard Collaborate con preguntas relacionadas con los informes de laboratorio elaborados por los alumnos (ver Figura 4). Una vez finalizada la evaluación, los alumnos pudieron visualizar las notas y los errores en las respuestas del examen. Finalmente se organizó una sesión final de evaluación para evaluar los resultados del proceso de enseñanza-aprendizaje.

En cuanto a la realización del examen, las profesoras de la asignatura prepararon un banco de preguntas con cuestiones previas (tipo test y de desarrollo) y problemas de cálculos numéricos. Se creó un banco de preguntas para las preguntas tipo test, otro para las preguntas de desarrollo y cinco más para los problemas de desarrollo. Estos bancos de preguntas se seleccionaron para la configuración del examen y se configuró la plataforma para que seleccionase preguntas en modo aleatorio, 5 tipo test, 2 de desarrollo y 2 problemas de cálculos numéricos. Los alumnos realizaron el examen online al mismo tiempo, estableciéndose un tiempo determinado. No se les permitía volver atrás para repasar las preguntas una vez contestadas. Con todas estas medidas se evitaron posibles comportamientos deshonestos por parte del alumno, lo que se puede aseverar en base a los comentarios realizados por los alumnos y las calificaciones numéricas obtenidas. 


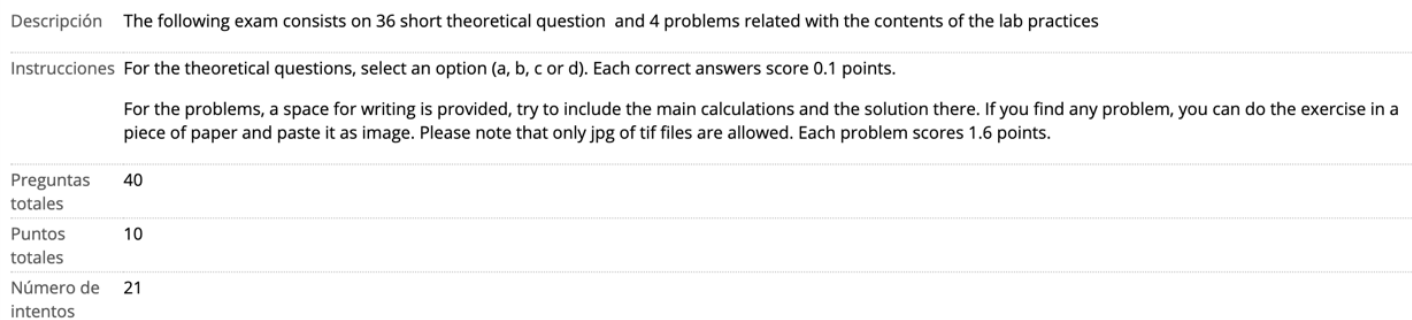

For the problems, a space for writing is provided, try to include the main calculations and the solution there. If you find any problem, you can do the exercise in a piece of paper and paste it as image. Please note that only jpg of tif files are allowed. Each problem scores 1.6 points.

$\square$ 1. Respuesta múltiple: The role of $\mathrm{BaCl} 2$ in the turbidimetri...

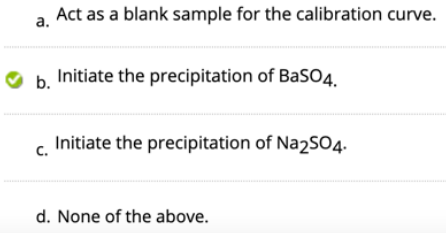

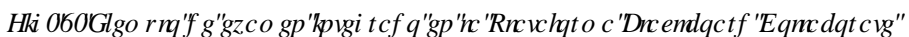

\section{Resultados}

El primer resultado evidente es el desarrollo de nuevas metodologías empleando en aprendizaje basado en problemas en el diseño de prácticas de laboratorio online para los alumnos de Farmacia. La experiencias obtenidas y aprendidas han servido como base para aplicar estas metodologías al modelo de enseñanza semipresencial adoptado en el curso 2020/2021.

Para evaluar la utilidad de la integración del aprendizaje basado en problemas, así como el impacto de la estrategia en el proceso enseñanza-aprendizaje, se realizó una breve encuesta de satisfacción a los alumnos, donde se les preguntó acerca del grado de satisfacción general. Como además realizaron las prácticas de forma presencial en el primer cuatrimestre se les pregunto por las posibles dificultades observadas en el aprendizaje en ambos formatos. El $90 \%$ de los alumnos se mostró muy satisfecho y no se constataron dificultades en el proceso de enseñanza-aprendizaje (ver Figura 5).
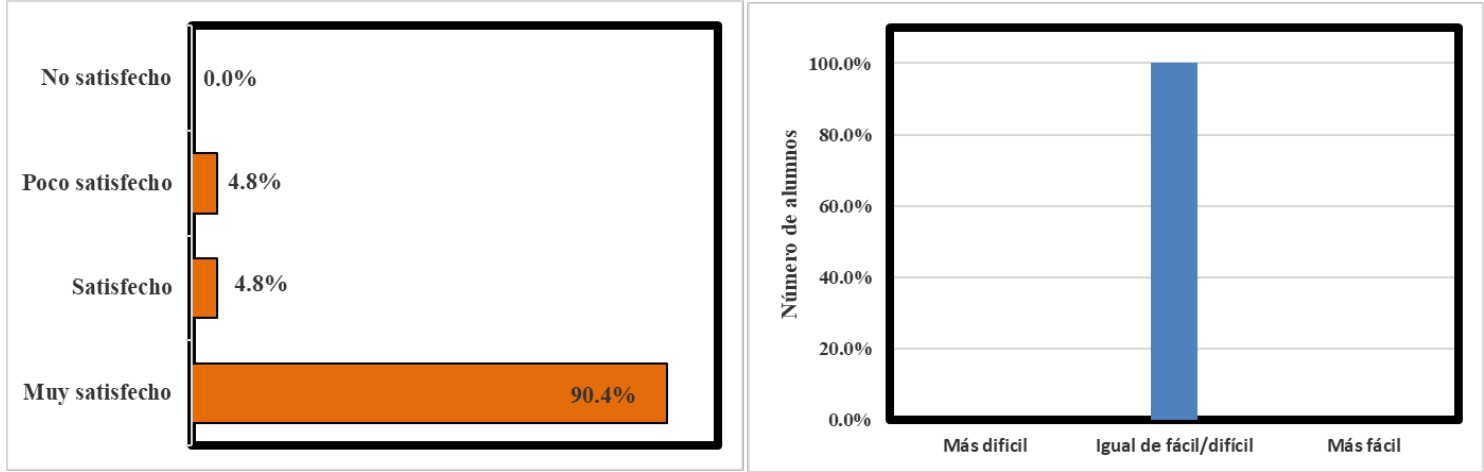

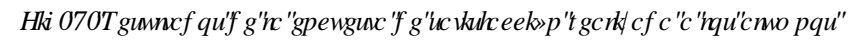

(c) EY-NC-ND 2021, Universitat Politècnica de València 
Adicionalmente, se compararon las calificaciones obtenidas con las que obtuvieron los alumnos en el año anterior en modelo presencial. Los resultados obtenidos se recogen en la Tabla 2.

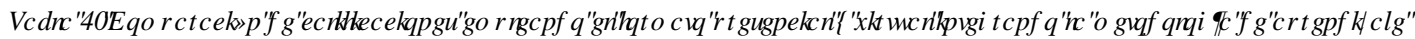
EDMORIHQSLRECP DV

\begin{tabular}{ccc}
\hline & $\begin{array}{c}\text { Curso 2018/2019 } \\
\text { Presencial }\end{array}$ & $\begin{array}{c}\text { Curso 2019/2020 } \\
\text { Online }\end{array}$ \\
\hline Número total de alumnos & 27 & 25 \\
Porcentaje aprobados & $100 \%$ & $70,4 \%$ \\
Número de alumnos no presentados & 0 & 4 \\
Resumen de calificaciones & Número de alumnos \\
Entre 0 y 4 & 11 & 1 \\
Entre 5 y 7 & 12 & 13 \\
Entre 7 y 10 & 4 & 7 \\
\hline
\end{tabular}

Como puede observarse, hay una gran diferencia en el porcentaje de aprobados. Mientras que en el formato on-line el $100 \%$ aprobó la asignatura, en el formato presencial este porcentaje fue de un 70,4\%. Cabe destacar aquí que en el Curso 2019/2020 no se presentaron 4 alumnos. Este hecho fue debido a que los alumnos repitieron la asignatura, pero en un curso académico anterior ya aprobaron las prácticas y se mantuvo su calificación ese año. Se puede concluir que la introducción de las metodologías de aprendizaje basadas en problemas aumentó el interés del alumno, favoreciendo la asimilación de competencias y conocimientos como se demuestra además en la mejora de las calificaciones, con un mayor número de alumnos obteniendo altas calificaciones. Hay que considerar aquí que el formato del examen fue distinto y evaluar el impacto de este hecho en las conclusiones.

\section{Conclusiones}

La pandemia de COVID-19 y la situación de confinamiento ha afectado en gran manera la actividad docente, requiriendo de nuevas metodologías de enseñanza remota no exploradas hasta la fecha. En especial, las prácticas de laboratorio en las áreas de ciencias experimentales requieren una participación activa del alumnado en el proceso de enseñanza aprendizaje para la adquisición de competencias, algo que no es posible realizar en el modelo de enseñanza remota.

En esta comunicación se ha descrito el empleo de nuevas metodologías de aprendizaje, en concreto el aprendizaje basado en problemas, para la docencia remota de prácticas de la asignatura "Analytical Techniques" del segundo curso del Grado en Farmacia de la Universidad de Alcalá. Se ha demostrado como se ha llevado a cabo la virtualización de la asignatura y la integración de esta metodología empleando la herramienta Blackboard Collaborate, vídeos explicativos de cada práctica de laboratorio y cuestionarios con cuestiones y datos ficticios equivalentes a los que los alumnos obtendrían en el laboratorio para llevar a cabo la resolución del caso/práctica de laboratorio. El proceso de enseñanza aprendizaje se evaluó mediante la calificación de los informes con hojas de rúbrica y un examen final. 
La evaluación del impacto de esta metodología y nuevo enfoque en la docencia se llevó a cabo mediante comparación de las calificaciones con las obtenidas en el modelo presencial y cuestionarios a los alumnos. El grado de satisfacción fue muy elevado (el $90 \%$ de los alumnos manifestó que estaba muy satisfecho con esta nueva metodología), con un porcentaje de aprobados del $100 \%$ frente al $70 \%$ obtenido en el modelo presencial en años anteriores. Se puede concluir que la introducción de las metodologías de aprendizaje basadas en problemas aumentó el interés del alumno, favoreciendo la asimilación de competencias y conocimientos como se demuestra además en la mejora de las calificaciones, con un mayor número de alumnos obteniendo altas calificaciones.

\section{Referencias}

ARPÍ, C., ÀVILA, P., BARALDÉS, M., BENITO, H., GUTIÉRREZ, Mª J., ORTS, M., ROSTAN, C.

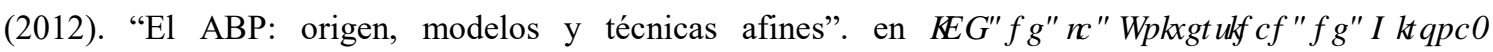
http://web2.udg.edu/ice/doc/xids/aula_educativa_1.pdf

BAO, W. (2020). "COVID-19 y la enseñanza en línea en la educación superior: un estudio de caso de la

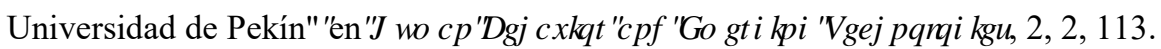

BARELL, J. (1999). "El aprendizaje basado en problemas. Un enfoque investigativo”. Manantial, ISBN 9875000310 .

GIL-GALVÁN, R. (2018). "El uso del aprendizaje basado en problemas en la enseñanza universitaria. Análisis de las competencias adquiridas y su impacto". en 5HLMD PHIFDQD GH IQYHWDDFyQ] HCXFDUMD, 23(76), 73-93.

HODGES, C., MOORE, S., LOCKEE, B., TRUST, T., Y BOND, A. (2020). "La diferencia entre la enseñanza remota de emergencia y el aprendizaje en línea." en (GXFDXVH $5 H M H Z$. https://er.educause.edu/articles/2020/3/the-difference-between-emergency-remote-teaching-and-onlinelearning.

LLORENS-MOLINA, J.Z. (2010). "El aprendizaje basado en problemas como estrategia para el cambio metodológico en los trabajos de laboratorio." en 4 XtP IFD1 RYD, 33, 994.

MARTINEZ, C., SALMERON, D., MORALES-DELGADO, N., ALONSO, A. (2020). "El aprendizaje basado en problemas como estrategia didáctica en el diseño de prácticas de Laboratorio Clínico y Biomédico" en 5HLLWD( ISDxRQD' H( GXFDFlyQ0 pGFD, 1, 105. 